\title{
Role of Contact and Contact Modification on Photo-response in a Charge Transfer Complex Single Nanowire Device
}

Rabaya Basori* and A. K. Raychaudhuri

(Received 28 October; accepted 15 December 2013; published online 11 January 2014)

\begin{abstract}
We investigated the feasibility of obtaining large photoresponse in metal-semiconductor-metal (MSM) type single nanowire device where one contact can be blocking type. We showed that suitable modification of the blocking contact by deposition of a capping metal using focused electron beam (FEB) can lead to considerable enhancement of the photoresponse. The work was done in a single Cu:TCNQ nanowire device fabricated by direct growth of nanowires (NW) from pre-patterned $\mathrm{Cu}$ electrode which makes the contact ohmic with the other contact made from Au. Analysis of the data shows that the large photoresponse of the devices arises predominantly due to reduction of the barriers at the $\mathrm{Au} / \mathrm{NW}$ blocking contact on illumination. This is caused by the diffusion of the photo generated carriers from the nanowires to the contact region. When the barrier height is further reduced by treating the contact with FEB deposited Pt, this results in a large enhancement in the device photoresponse.
\end{abstract}

Keywords: Nanowire; Cu:TCNQ; MSM device; FEB deposition; Photo-response

Citation: Rabaya Basori and A. K. Raychaudhuri, "Role of Contact and Contact Modification on Photoresponse in a Charge Transfer Complex Single Nanowire Device", Nano-Micro Lett. 6(1), 63-69 (2014). http:// dx. doi.org/10.5101/nml.v6i1.p63-69

\section{Introduction}

Nanotubes, nanowires and nanobelts are important one-dimensional (1D) nanomaterials that are the foundation for nanoscience and nanotechnology [1-14]. Novel nanoscale optoelectronic devices are important applications of nanomaterials, particularly nanowires which can be scaled down to a single nanowire (NW) device [1-12]. Contact electrodes play an important role in improving performance of nanoscale devices [1$2]$. For metal-semiconductor (MS) contacts there are mainly two different types of contacts: Ohmic contact and blocking or rectifying contacts. In the first type of contact work function of semiconductor is larger than metal and electrons are transferred from metal to semiconductor for n-type material, producing an accumulation layer in the semiconductor. In the blocking con- tacts, work function of metal is larger than the semiconductor and electrons are transferred from semiconductor to metal for n-type material, leaving a depletion layer in the semiconductor and leading to formation of a barrier. The role of contact becomes especially important in the context of photoresponse in nanowires. In opto-electronic devices based on nanowires like solar cell [3], nanowire sensors [4], photodetector [5] etc. The crucial parameter is often the photoconductive gain $G$, defined as $G \equiv I_{\mathrm{ph}} / q F$, where $I_{\mathrm{ph}}$ is the photocurrent generated due to absorbed photon flux $F, q$ being the charge. In general a photoconductive gain $G>1$ is seen in devices with good Ohmic contact at the metal-semiconductor interface. It is generally believed that in photo detectors with blocking contact at the metal-semiconductor junction, it is not possible to obtain a photoconductive gain $G>1[6-8]$. As a re-

Theme Unit of Excellence in Nano Device Technology and Department of Condensed Matter Physics and Materials Science, S.N. Bose National Centre for Basic Sciences, Salt Lake, Kolkata-700098, India

*Corresponding author. E-mail: rabaya@bose.res.in, arup@bose.res.in 
sult, positive effect of non-ohmic or blocking contact (like a Schottky contact) has not received much attention in optoelectronic devices, despite observation of increase photoconductive gain $G$ in some photo-detectors. In recent years, in context of nanowires, this question has gained significance. In earlier work, different materials have been used as photoconductive device e.g. $\mathrm{MoS}_{2}$ [1], Si [3], $\mathrm{ZnO}$ [9], $\mathrm{Bi}_{2} \mathrm{~S}_{3}$ [10], InP [11], CdS [12] etc. It is reported that these material can give substantial photoconductive gain using Schottky contact where they have been used as single nanowire device. Recently we have shown that nanobridges made from an array of nanowire of a charge transfer complex $\mathrm{Cu}$ :TCNQ can show large photoresponse in a MetalSemiconductor-Metal (MSM) device configuration [15]. It was argued that lowering of barrier due to diffusion of photo-generated carriers in the contact region can lower the barrier thus enhancing the photocurrent. It is expected that in nanowire devices that show large photoresponse, the contact will play an important role and its suitable modification can lead to enhancement of the photo-response.

In this paper we investigate the role of contact in photo response where the contact barrier can be modified after the device is fabricated using focused ion beam (FIB) or focused electron beam (FEB) deposited metal. Though FIB or FEB deposited metals like Pt or $\mathrm{W}$ have been used to mend electrical circuits, their use to modify contact barriers to engineer the response in single nanowires optical devices have not been demonstrated. Using a single nanowire device where one of the contacts is blocking type and other Ohmic, we establish the role of the blocking contact and its modification by FIB or FEB deposited metal in determination of the photoresponse of such a single nanowire device.

For this work we had made a single nanowire device from charge transfer complex Cu:TCNQ with one probe Ohmic and another probe blocking (Schottky) type. The reason for choosing this material is that it shows large photoresponse where the barrier plays an important role [15] and in this material by choice we can make one of the junctions Ohmic using a growth protocol described below. This particular choice may not be available in many other materials. After the device is fabricated and measurements is performed in dark and under illumination, the blocking contact is then modified with Focused Electron Beam induced deposited (FEBID) Pt. The resulting change in the device response, both in dark and under illumination, has been analyzed to investigate the effect of contacts on the photoresponse of such a device. Though the experiment has been carried out in the specific context of a charge transfer nanowire it is expected to have a general implication for single nanowires devices where such barrier modification can indeed enhance the current under illumination. (We note that in the context of the charge transfer complex $\mathrm{Cu}$ :TCNQ, effect of contacts have been investigated in the context of resistive state switching [16]. However, no report has investigated the specific role of contact and its modifications in regard to photoresponse in such a material).

\section{Experimental}

The device is fabricated using in-situ growth of nanowires within prefabricated metal electrodes by physical vapor deposition method. This method allows growth of nanowires at predetermind sites. Nanowires are characterized by SEM, FTIR, Raman, X-RD. The as grown nanowires are further characterized by TEM and selected area electron diffraction (SAED) patternsas shown in $(\mathrm{g})$ of Fig. 1(A) bottom frame, which proves that all of these nanowires are crystalline in nature. Use of this growth protocol is novel because the $\mathrm{Cu}$ from which the growth occurs serves as the Ohmic contact and no other contact fabrication is needed. The process used for making the electrodes and the arrangement of growth from vapour phase is shown in Fig. 1(A) and 1(B) respectively. The wires are laterally grown from $\mathrm{Cu}$ after reacting with vapour of TCNQ obtained from a boat (kept at $130^{\circ} \mathrm{C}$ ) containing TCNQ powder on prefabricated bi-layer $\mathrm{Cu} / \mathrm{Au}$ electrodes where $\mathrm{Cu}$ acts as the bottom electrode and $\mathrm{Au}$ as top electrode. The bi-layer electrodes with typical separation $\approx 1.0$ $\mu \mathrm{m}$ are made by electron beam lithography and lift-off process on $\mathrm{SiO}_{2} / \mathrm{Si}$ substrates (as shown schematically in Fig. $1(\mathrm{~A})$ ) where the top oxide layer $\approx 300 \mathrm{~nm}$. The vapour deposition is done by keeping the substrate in a certain angle with respect to the vapour in such a way that nanowires start to grow from the $\mathrm{Cu}$ part of one electrode and terminate on the top Au layer of the second electrode, thus completing the circuit. The typical diameter of the nanowires in the device is $\approx 30 \mathrm{~nm}$. The FE-SEM (Field-Emission Scanning Electron Microscopy) image of the device (marked as S1) is shown in Fig. 1(A) bottom frame. This is a single NW device. The device so formed has a $\mathrm{Cu}$ electrode that we will show is Ohmic and an Au electrode that is blocking type with higher barrier. (We provide an estimate of barriers from measurements later on). After measurement of the $I$ - $V$ curves in dark and under illumination and the optical response under zero bias, the Au electrode, which forms the high barrier contact, was modified by capping it with FEBID Pt. For capping free end of the nanowire with $\mathrm{Au}$ electrode in a dual beam machine FEI HELIOS 600, the wire is imaged using FE-SEM (Field-emission scanning electron microscope) and after selecting the specified single nanowire free end is then anchored with $\mathrm{Pt}$ deposited by the electron beam of the FE-SEM at a voltage of $15 \mathrm{keV}$ and deposition current of $90 \mathrm{pA}$ using the precursor Methylcyclopen- 


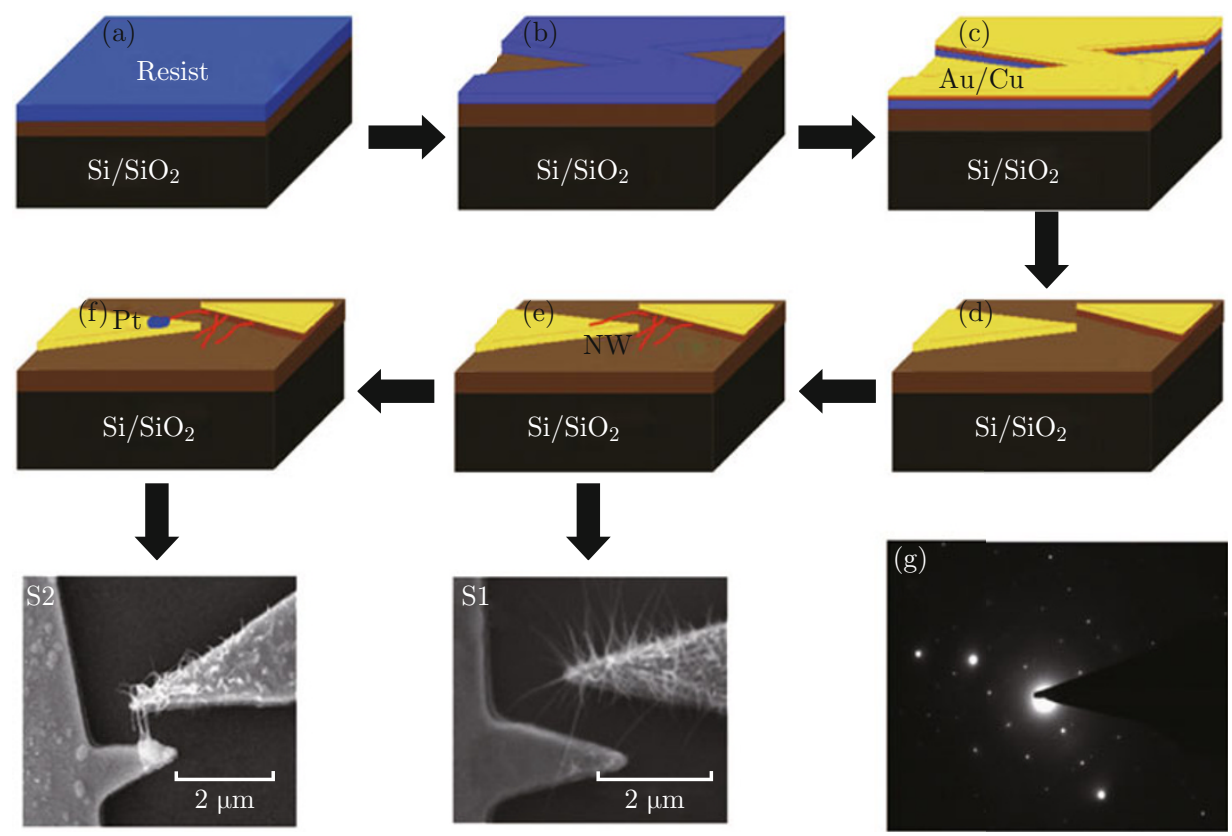

(A)
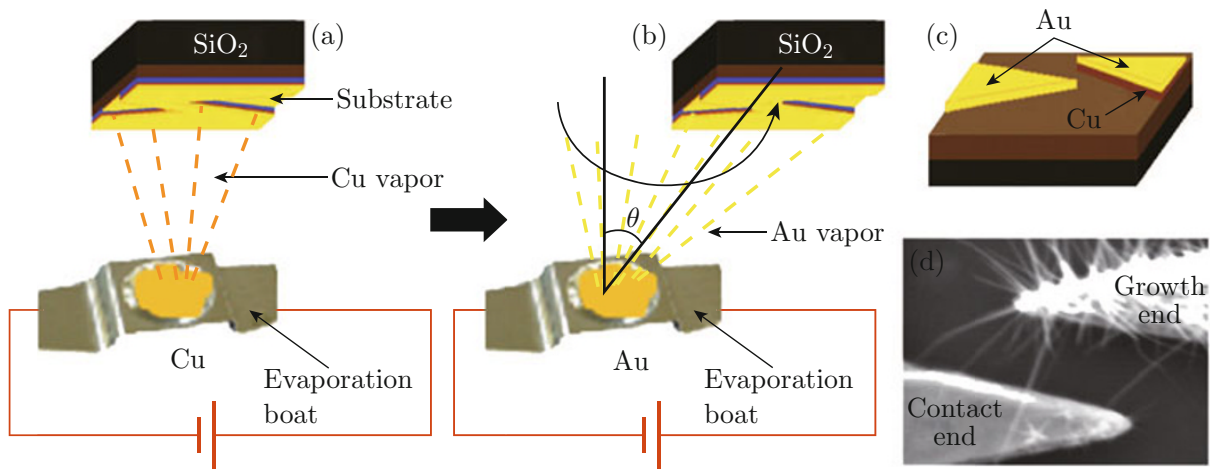

(B)

Fig. 1 (A) Schematic representation of procedure of making of the devices: (a) Resist coated $\mathrm{Si}_{\mathrm{SiO}}$; (b) Pattern written by NPGS (Nano Pattern Generating System) software; (c) Au/Cu evaporation; (d) Lift-off; (e) Growth of nanowire on predefined pattern; (f) Anchoring on top of free end of the nanowire with FEB deposited Pt; and (g) SAED pattern of one such nanowire. S1 and S2 are SEM images of the nanowire devices before and after anchoring of free end of the nanowire respectively. (B) Schematic representation of procedure of making growth of the nanowires within the prefabricated pattern: (a) During $\mathrm{Cu}$ deposition sample was on top of $\mathrm{Cu}$ evaporation boat; (b) Au was deposited rotating the sample holder by an angle $\theta$ w.r.t. the Au source in such a way that $\mathrm{Au}$ vapour will come direct contact to one electrode pattern and shadow effect arise to the rest; (c) This results one $\mathrm{Cu}$ electrode fully covered with $\mathrm{Au}$ and another $\mathrm{Cu}$ electrode is partially covered with $\mathrm{Au}$; and (d) SEM image of lateral growth of the Cu:TCNQ nanowire starts from $\mathrm{Cu}$ and connect to Au electrode.

tadienyl platinum trimethyl $\left(\mathrm{CH}_{3}\right)_{3}\left(\mathrm{CH}_{3} \mathrm{C}_{5} \mathrm{H}_{4}\right)$ Pt. The FEBID does not substantial cause the damage that is generally associated with Focused Ion Beam induced deposition (FIBID) of $\mathrm{Pt}$ which is done using Ga ions. However, there may be a small degradation of the NW resistance on exposure to the electron beam during the deposition. This device, with $\mathrm{Pt}$ deposited modification is marked as S2 and its FE-SEM image is shown in Fig. 1(A) bottom frame. All other parameters, including the NW being essentially unchanged, this process allows us to evaluate the explicit role of modifying the $\mathrm{Au}-\mathrm{NW}$ contact by $\mathrm{Pt}$ capping.

Optical excitation used in this work is provided by
Helium lamp (450 W) and a monochromator. Bias dependent dark and photocurrent is measured using a Sourcemeter (Keithley 2400) and a Picoammeter (Keithley 485). All the data are taken under illumination of light of wavelength $\sim 405 \mathrm{~nm}$ because the device is found to show maximum photoresponse at that wavelength (data not shown).

\section{Results and discussions}

The $I-V$ data in dark as shown in Fig. 2 is for the device before and after modification of the blocking con- 
tact. The positive bias refers of the $\mathrm{Cu}$ electrode between positive from which the growth starts (marked in Fig. 1(B)). It is seen that after anchoring (capping the $\mathrm{Cu}: \mathrm{TCNQ} / \mathrm{Au}$ contact with FEB deposited Pt) there is an enhancement of the device current. This enhancement mainly arises from the reduction of the barrier height at the blocking contact as the analysis shown below. The $I-V$ data is analyzed in the frame work of MSM configuration where the $\mathrm{Cu}$ and the $\mathrm{Au}$ electrodes connecting the nanowire act as the metal (M) electrodes and $\mathrm{Cu}$ TCNQ nanowire is the semiconductor. The bias dependent current through the device is fitted to the model of two back to back Schottky diodes connected by a series resistance $R$, which mainly represents the resistance due to the portion of the NW between the electrodes. The equation for fit used is [17]:

$$
\begin{aligned}
I(V)= & I_{0} \exp \left(\frac{q V^{\prime}}{\eta k T}-1\right) \cdot \\
& \frac{\exp \left(\frac{-q\left(\phi_{1}+\phi_{2}\right)}{k T}\right)}{\exp \left(\frac{-q \phi_{2}}{k T}\right)+\exp \left(\frac{-q \phi_{1}}{k T}\right) \exp \left(\frac{q V^{\prime}}{\eta k T}\right)}
\end{aligned}
$$

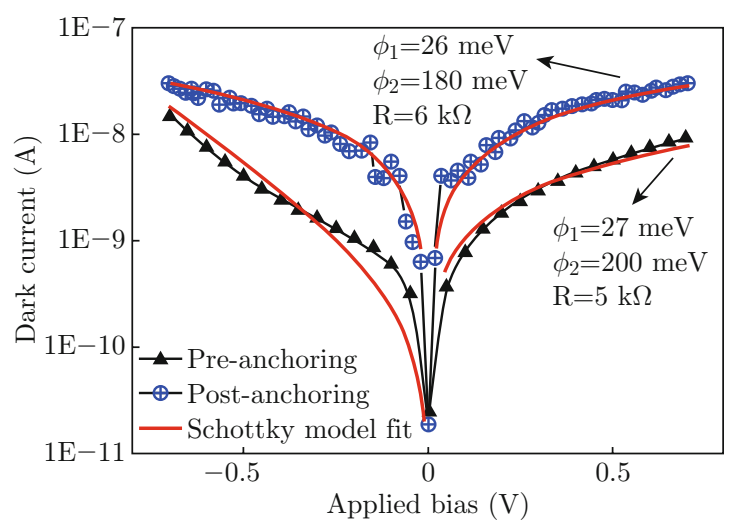

Fig. $2 I-V$ curves of Cu:TCNQ nanowire MSM devices in dark. As prepared device (marked as pre-anchoring/S1) and that after anchoring the Au contact by FEB deposited $\mathrm{Pt}$ with $\mathrm{Au}$ electrode (marked as post-anchoring/S2). The barrier heights $\phi_{1}$ and $\phi_{2}$ for the device S1 obtained from model fit are $\phi_{1}=26 \mathrm{meV}, \phi_{2}=200 \mathrm{meV}$ and those for device $\mathrm{S} 2$ are $\phi_{1}=27 \mathrm{meV}, \phi_{2}=180 \mathrm{meV}$.

where, $V^{\prime}=V-I R, R$ being the series resistance which is mainly the resistance of the nanowire, and $\phi_{1}$ and $\phi_{2}$ are the barrier heights associated with two contacts (M's). $I_{0}$ the current due to thermionic emission and is used as a constant in the fit process. Barrier $\phi_{1}$ refers to the $\mathrm{Cu}$ electrode and barrier $\phi_{2}$ to the $\mathrm{Au}$ electrode. From the fit of the experimental dark $I-V$ data to equation 1 , we obtain $\phi_{1} \approx 27 \mathrm{meV}$ and $\phi_{2} \approx 200 \mathrm{meV}, R=$ $5 \mathrm{k} \Omega$ (In the fit procedure $R$ has been taken as a fit parameter along with $\phi_{1}$ and $\left.\phi_{2}\right)$. Solid lines through the data in Fig. 2 show the fit to the $I-V$ curves. As a validation of the model we find that the value of $R$ obtained from the model fit is close to the value calculated from the independently measured 4-probe resistivity of the nanowire. The asymmetry in the $I-V$ data can be explained by a difference in the work functions at the two electrodes. The value of the barrier $\phi_{1} \approx 27 \mathrm{meV}$ for the $\mathrm{Cu} / \mathrm{NW}$ contact, from where the growth of the wire starts, is low enough at room temperature $(\sim 25 \mathrm{meV})$ so that it can be considered as nearly Ohmic. However, for the $\mathrm{Au} / \mathrm{NW}$ contact the barrier $\phi_{2} \approx 200 \mathrm{meV}$ is substantial and it acts as the blocking electrode. Use of the $\mathrm{Cu}$ from which the growth occurs as an Ohmic electrode is advantageous because it reduces the fabrication steps.

In Fig. 2 we also show the $I-V$ curve for the device $\mathrm{S} 2$, after capping the free end of the nanowire with $\mathrm{Au}$ electrode by FEB deposited Pt. From analysis of the $I-V$ curve as done above, we find that $\phi_{2}$ is reduced to $180 \mathrm{meV}$ while the other electrode (Ohmic contact) $\phi_{1}$ stays unchanged as this was not modified. The resistance of the NW $R$ changes somewhat during the capping process and increases by $20 \%(6.0 \mathrm{k} \Omega)$ as shown in Fig. 2. The reduction of the barrier height $\left(\phi_{2}\right)$ after modification of the contact may be due to change in local work function and bend alignment. Since barrier height change occurs in the exponential its reduction has substantial effect.

The effect of the contact and the contact modification on the photoresponse was investigated. In Fig. 3 we show the results of measurement of the photoresponse. The data shown are for zero bias (applied bias $V=$ 0 ). As shown in Fig. 3, the zero bias photo-response, which is dominated by contact barriers, is enhanced significantly in device S2 due to the capping by Pt. Photocurrent as a function of bias for both the cases (pre and post anchored) is shown in Fig. 4. The current shown is the total device current measured, which

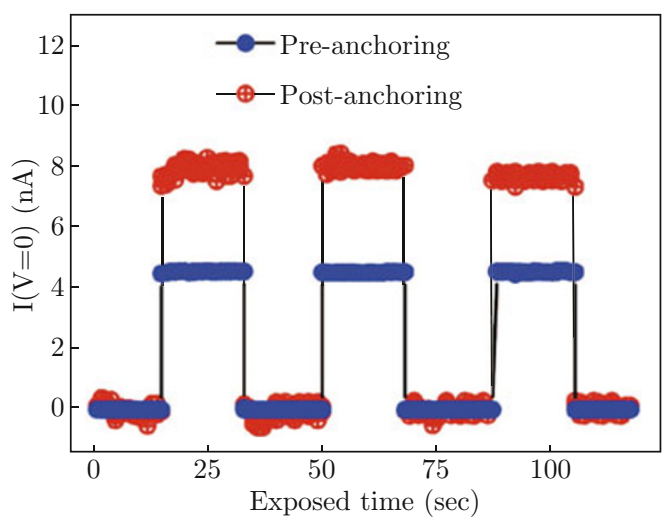

Fig. 3 Zero bias photo-response (applied bias $V=0$ ) of the single nanowire devices. The enhancement in response before (S1) and after anchoring (S2) can be seen. Data were taken with illumination at $\lambda=405 \mathrm{~nm}$. 


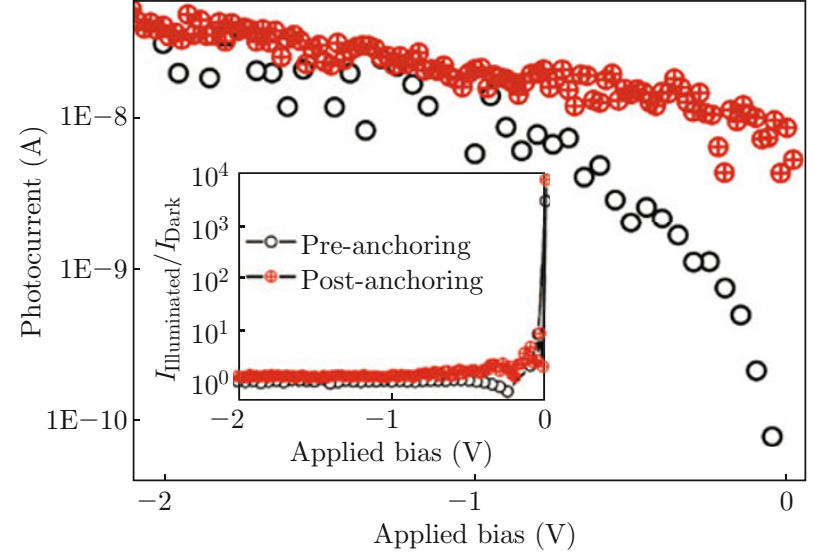

Fig. 4 Bias dependence of the photo current $I_{p h}=$ $I_{i l l}-I_{\text {dark }}$ as a function of reverse bias. Data were taken with illumination at $\lambda=405 \mathrm{~nm}$. The inset shows the bias dependence of the ratio $I_{i l l} / I_{\text {dark }}$.

under illumination is marked as $I_{i l l}$ while that under dark is the dark current $I_{\text {dark }}$. (The bias dependence of the of the ratio $I_{i l l} / I_{\text {dark }}$ is shown in inset of Fig. 4 for the two devices.) The photocurrent $I_{p h}(V)$ at an applied bias $V$ is defined as difference of the current under illumination $I_{i l l}$ and the dark current $I_{d a r k}$ at a given bias $\left(I_{p h} \equiv I_{i l l}-I_{\text {dark }}\right)$. There is no external field at zero bias and photogenerated carriers reach the electrodes without any recombination. This is possibly because of the close proximity of the electrodes $\sim 1$ $\mu \mathrm{m}$, which is smaller than or of the order of the typical recombination length in such materials.

The enhancement of the photocurrent after the barrier modification mainly occurs at the low bias as one can see in Fig. 4. This is expected because at higher bias the device current is restricted by the wire resistance due to finite voltage drop across it. In the low bias region, the device current is mainly junction limited being controlled by the barrier height. This can also be seen in the inset of Fig. 4, where we plot the ratio $I_{i l l} / I_{\text {dark }}$ as a function of the bias. It can be seen that at higher bias $(|V| \geq 0.5 \mathrm{~V})$ the effect of barrier modification tapers off as effect of the illumination also tapers off.

From the measured $I-V$ curve under illumination, using Eqn. (1), we could deduce the barrier height modifications produced by the illumination. Both the barrier heights change to lower values. For instance for device $\mathrm{S} 1, \phi_{1} \approx 20 \mathrm{meV}$ and $\phi_{2} \approx 160 \mathrm{meV}$ and the resistance of the nanowires reduces to $R=0.9 \mathrm{k} \Omega$ under illumination. The change in barrier heights on illumination are $\Delta \phi_{1} \approx 7 \mathrm{meV}$ and $\Delta \phi_{2} \approx 40 \mathrm{meV}$ with a reduction in resistance by a factor of $1 / 5$ due to photoconductivity. The photosensitivity $S$ defined as $\mathrm{S} \equiv$ $\left(\sigma_{p h}-\sigma_{\text {dark }}\right) / \sigma_{\text {dark }}$, where $\sigma_{p h}\left(\sigma_{\text {dark }}\right)$ is the conductivity under illumination (dark) can be evaluated from the change in resistance and for the device $\mathrm{S} 1, S \approx 4.6$. After modification of contact, in S2, the barrier height $\phi_{2} \approx 130 \mathrm{meV}$ under illumination, corresponding to a change of $\Delta \phi_{2} \approx 50 \mathrm{meV}$. The change in $\Delta \phi_{1}$ is similar to that in S1. Under illumination in S2 nanowire resistance reduces from $R=6.0 \mathrm{k} \Omega$ to $R=0.5 \mathrm{k} \Omega$ leading to a photosensitivity $S \approx 11$. It appears that the exposure to the electron beam during deposition may lead to enhancement of the photosensitivity $S$ although this being beyond the scope of the investigation was not investigated.

The enhancement of the total photocurrent in the single nanowires MSM device has contributions of illumination-induced reduction of the barrier at the junction, as well as photoconductive reduction of the series resistance of the wire which gives us a measure of the photosensitivity $S$. In the present device the lowering of the barrier at the contact under illumination is a major reason for the photo response, which in turn accentuates the effect of the photoconductivity. As a result the barrier modification leads to a change in current in the device. Importantly, the barrier modification has a large effect on the zero bias photocurrent $I_{\mathrm{ph}}(V=0)$ as can be seen from Fig. 3. The enhancement of the photo current can be nearly 2 orders for $V=0$. The change in the photoconductivity being much less, it is the modification of the contact by the FEB deposited Pt which causes this large change. All the measurements are done at low bias so that the nanowire is under safe region of electric field below the phase change threshold during the measurements [18]. We could also measure the bias driven resistive state transition in such wires (length $\approx 1.5-2.0 \mu \mathrm{m}$ ) occurring at a bias of $\approx 3 \mathrm{~V}$ corresponding to a field of $(1.5-2.0) \times 10^{6} \mathrm{~V} / \mathrm{m}$ (Data not shown). This shows that even at the highest optical power density used, the electric field of the light used (at $\lambda=405 \mathrm{~nm}) E_{\text {opt }}\left(\sim 7 \times 10^{4} \mathrm{~V} / \mathrm{m}\right)$ is much less than the applied electric field $E_{\text {switch }}\left(\geq 2 \times 10^{6} \mathrm{~V} / \mathrm{m}\right)$ needed to switch the resistive state to a low resistance state.

The lowering of barrier on illumination occurs because a large number of carriers that are created due to illumination diffuse into the contact region. Control of contact barrier by electrical gate controlled charge injection has been recently utilized in Si NW devices [19]. An estimate of the barrier lowering $\Delta \phi$ can be obtained from the change in the chemical potential due to change in the carrier density $\Delta n$ on illumination and is given as:

$$
\Delta \varphi \approx \frac{k_{B} T}{q} \ln \left(\frac{n_{0}+\Delta n}{n_{0}}\right)
$$

where $n_{0}$ is the carrier density in the dark [20]. To estimate $\Delta n$ we use the change in the photoconductivity of the nanowires as derived from the analysis above. If the change in the conductivity due to the illumination $\left(\Delta \sigma=\sigma_{p h}-\sigma_{d a r k}\right)$ of the NW is solely due to the change in the carrier density $(\Delta n)$, with no change in 

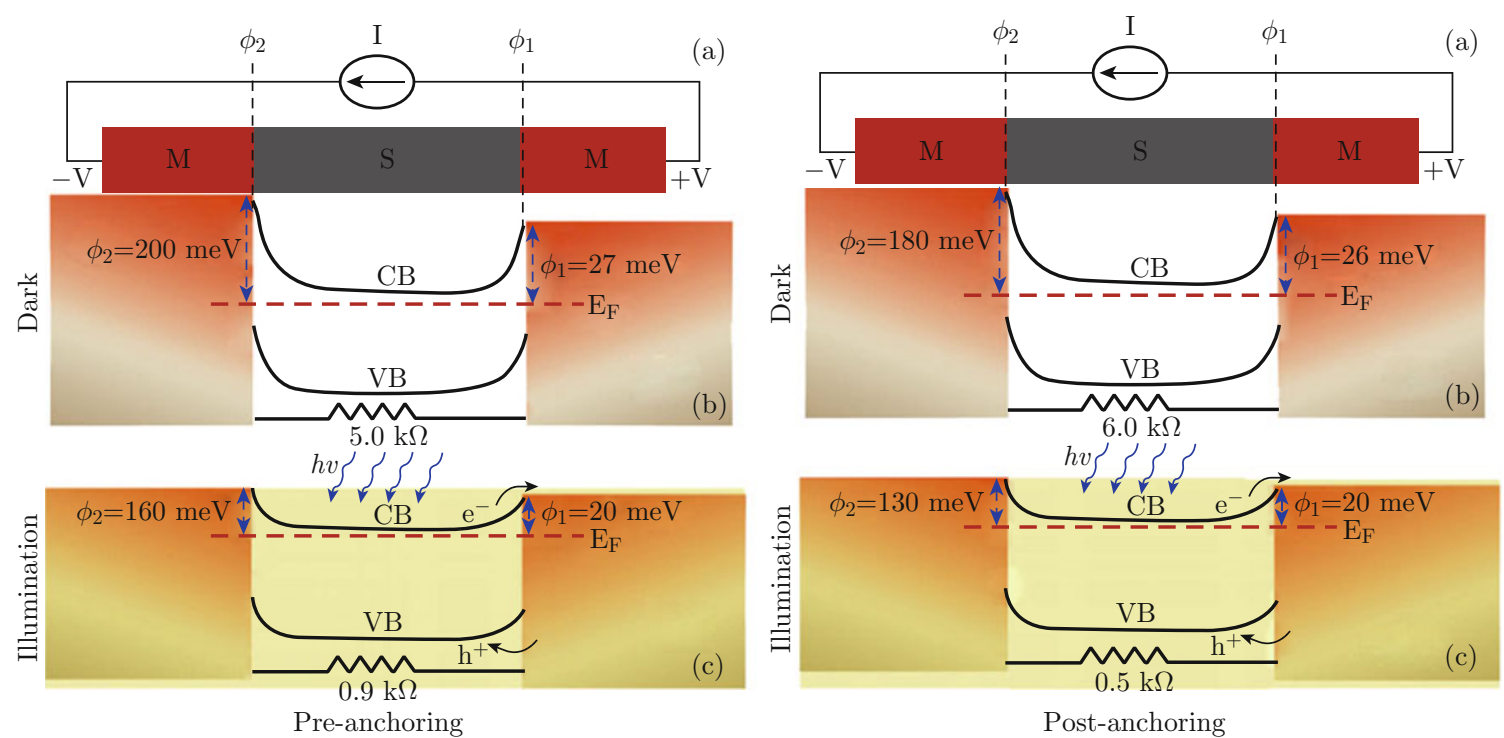

Fig. 5 Comparative study of the parameters for nanowire MSM devices before (left) and after (right) Pt deposition: (a) Schematic structure with barrier heights derived from model with illumination and in dark; (b) Band bending of the conduction band in dark and (c) Band bending under illumination. Relevant barrier heights as well as the resistances of the strand of the nanowires for the two devices are shown as derived from the model in dark and under illumination.

the mobility, then we get a measure of the change in carrier density from the observed $\mathrm{S}$. $\mathrm{S} \equiv\left(\sigma_{p h}-\right.$ $\left.\sigma_{\text {dark }}\right) / \sigma_{\text {dark }}=\Delta n / n_{0}$, where $n_{0}$ is the carrier density in the dark. For device $\mathrm{S} 1$, from observed $S \approx 4.6$, we estimate shift in the barrier $\Delta \phi \approx 43 \mathrm{meV}$ on illumination. This is close to the shift of $40 \mathrm{meV}$ observed from experiments. After Pt deposition and the barrier modification in device S2, the observed photosensitivity $S$ reaches a value $\approx 11$. This would give rise to an estimate shift in the barrier $\Delta \phi \approx 62 \mathrm{meV}$. This is somewhat larger but close to the observed shift of $50 \mathrm{meV}$. The $\mathrm{Cu}$ contact being Ohmic with a low enough barrier, there is a negligible change in the barrier height.

In both the devices studied we find clear signature of photocurrent at zero bias that is substantially larger than the dark current and the current critically depends on the barrier height and the nature of the contact. The observation of zero bias current in both the devices (S1 and S2) points to a common origin. The existence of such a current depends on the field due to the built-in potential that exists near the contacts. If the electrode separation is more than the depletion width, there will be no axial field in the middle of the NW in absence of an applied bias. In this case the photo-generated carriers will not reach the electrodes. However, in our case the electrode separation being small it is likely that it is less than the total of depletion widths at the contact regions. This will give rise to a built-in field which will collect the carriers. If the two junctions are symmetrical then the net current at zero bias will be zero. However, existence of asymmetric junctions at the two ends will ensure a non-zero net current. Another mechanism that also contributes to the transport of photo-generated carriers in MSM junction is tunnel- ing through the barrier at the junction [21]. This is prevalent when the photon energy $h v$ is much greater than the barrier height $\phi$, which is adequately satisfied in our case. Existence of such a tunneling of the photoelectron at the junction can thus be another source of the photocurrent at zero bias. The nature of the contact is thus a critical parameter for collection of charge and results in a zero bias current. It is then justified that the barrier modification by FEB deposited Pt that modifies the barrier height will affect this process. The barrier height reduction due to the capping of the contact will thus enhance the photo current.

The physical process that leads to the dominant role of the contact and the contact modification can be best summarized by the schematic band diagram shown in Fig. 5. The figure shows the barriers, the depletion regions, the changes in these parameters in dark and under illumination in both the devices that lead to the changed response on modification of the contact.

\section{Conclusion}

In summary, we have demonstrated that it is possible to obtain large photoresponse in MSM type device where one contact can be blocking type. We further showed that suitable modification of the blocking contact can lead to further enhancement of the photoresponse. This was done in the context of a single $\mathrm{Cu}:$ TCNQ nanowire device. We demonstrated that this type of device can be fabricated by direct growth of nanowires from pre-patterned $\mathrm{Cu}$ electrode which leads to one contact ohmic. Analysis of the $I$ - $V$ curves in dark and under illumination with MSM Schottky diode 
model shows that the large photoresponse of the devices arise predominantly due to reduction of the Schottky barriers at the MS interfaces due to diffusion of the photo generated carriers from the NW to the contact region. Barrier height of Schottky contact is further reduced by treating the contact with FEB deposited $\mathrm{Pt}$ resulting in a large enhancement in photoresponse even in zero bias due to combine effect of barrier height reduction and increase in charge carrier generation.

\section{Acknowledgements}

The authors thank Nanomission, Department of Science and Technology, Govt. of India for financial support as sponsored project: Theme Unit of Excellence in Nanodevice Technology. AKR wants to thank J. C. Bose fellowship for additional support.

\section{References}

[1] M. Fontana, Deppe T, A. K. Boyd, M. Rinzan, A. Y. Liu, M. Paranjape and P. Barbara, "Electron-hole transport and photovoltaic effect in gated $\mathrm{MoS}_{2}$ Schottky junctions", Sci. Rep. 3, 1634-4 (2013). http://dx. doi.org/10.1038/srep01634

[2] E. J. H. Lee, K. Balasubramanian, R. T. Weitz, M. Burghard and K. Kern, "Contact and edge effects in graphene devices", Nat. Nanotechnol. 3(8), 486-490 (2008). http://dx.doi.org/10.1038/nnano. 2008. 172

[3] Y. Ahn, J. Dunning and J. Park, "Scanning photocurrent imaging and electronic band studies in silicon nanowire field effect transistors", Nano Lett. 5(7), 1367-1370 (2005). http://dx.doi.org/10.1021/ $\mathrm{n} 1050631 \mathrm{x}$

[4] R. Yu, C. Pan, J. Chen, G. Zhu and Z. L. Wang, "Enhanced performance of a $\mathrm{ZnO}$ nanowirebased self-powered glucose sensor by piezotronic effect", Adv. Funct. Mater. 23(5), 5868-5874 (2013). http://dx.doi.org/10.1002/adfm. 201300593

[5] Y. Liu, Q. Yang, Y. Zhang, Z. Yang and Z. L. Wang, "Nanowire piezo-phototronic photodetector: Theory and experimental design", Adv. Mater. 24(11), 1410-1417 (2012). http://dx.doi.org/10. 1002/adma. 201104333

[6] R. H. Bube: Photoconductivity of Solids (Wiley, New York, 1960).

[7] Rose: Concepts in Photoconductivity and Allied Problems (Interscience, New York, 1963).

[8] W. Ruppel: II-VI Semiconducting Compounds, edited by D. G. Thomas (Benjamin, New York, 1967).

[9] Y. Hu, J. Zhou, P. H. Yeh, Z. Li, T. Y. Wei and Z. L. Wang, "Supersensitive, fast-response nanowire sensors by using Schottky contacts", Adv. Mater. 22(30), 3327-3332 (2010). http://dx.doi.org/10. 1002/adma. 201000278

[10] H. Bao, X. Cui, C. M. Li, Y. Gan, J. Zhang and J. Guo, "Photoswitchable semiconductor Bis- muth Sulfide $\left(\mathrm{Bi}_{2} \mathrm{~S}_{3}\right)$ nanowires and their selfsupported nanowire arrays", J. Phys. Chem. C 111(33), 12279-12283 (2007). http://dx.doi.org/10. 1021/jp073504t

[11] A. Maharjan, K. Pemasiri, P. Kumar, A. Wade, L. M. Smith, H. E. Jackson, J. M. Yarrison-Rice, A. Kogan, S. Paiman, Q. Gao, H. H. Tan and C. Jagadish, "Room temperature photocurrent spectroscopy of single zincblende and wurtzite InP nanowires", Appl. Phys. Lett. 94(19), 193115 (2009). http://dx.doi. org/10.1063/1.3138137

[12] Y. Gu, E-S. Kwak, J-L. Lensch, J-E. Allen and T-W Odom, "Near-field scanning photocurrent microscopy of a nanowire photodetector", Appl. Phys. Lett. 87(4), 043111 (2005). http://dx.doi.org/10. 1063/1. 1996851

[13] J. Xiao, Z. Yin, H. Li, Q. Zhang, F. Boey, H. Zhang and Q. Zhang, "Postchemistry of organic particles: when TTF microparticles meet TCNQ microstructures in aqueous solution", J. Am. Chem. Soc. 132(20), 69266928 (2010). http://dx.doi.org/10.1021/ja102154b

[14] J. Xiao, H. Yang, Z. Yin, J. Guo, F. Boey, H. Zhang and Q. Zhang, "Preparation, characterization, and photoswitching/light-emitting behaviors of coronene nanowires", J. Mater. Chem. 21(5), 1423-1427 (2011). http://dx.doi.org/10.1039/c0jm02350g

[15] R. Basori, K. Das, P. Kumar, K. S. Narayan and A. K. Raychaudhuri, "Large photoresponse of $\mathrm{Cu}: 7,7,8,8$ tetracyanoquinodimethane nanowire arrays formed as aligned nanobridges", Appl. Phys. Lett. 102(6), 061111 (2013). http://dx.doi.org/10.1063/1.4792470

[16] A. Hefczyc, L. Beckmann, E. Becker, H-H. Johannes and W. Kowalsky, "Contact effect in $\mathrm{Cu}$ (TCNQ) memory devices", Phys. Status Solidi A 205(3), 647-655 (2008). http://dx.doi.org/10.1002/pssa. 200723418

[17] H. Norde, "A modified forward IV plot for Schottky diodes with high series resistance", J. Appl. Phys. 50(7), 5052-5053 (1979). http://dx.doi.org/ $10.1063 / 1.325607$

[18] K. B. Zheng, H. T. Shen, C. N. Ye, J. L. Li, D. L. Sun and G. R. Chen, "The electrical switching characteristics of single copper tetra-cyanoquinodimethane nanowire", Nano-Micro Lett. 1(1), 23-26 (2009). http://dx.doi.org/10.5101/nml.v1i1.p23-26

[19] M. Mongillo, P. Spathis, G. Katsaros, P. Gentile and S. D. Franceschi, "Multifunctional devices and logic gates with undoped silicon nanowires", Nano Lett. 12(7), 3074-3079 (2012). http://dx.doi.org/ $10.1021 / \mathrm{nl} 300930 \mathrm{~m}$

[20] A. Zhang, H. Kim, J. Cheng and Y-H. Lo, "Ultrahigh responsivity visible and infrared detection using silicon nanowire phototransistors", Nano Lett. 10(6), 21172120 (2010). http://dx.doi.org/10.1021/nl1006432

[21] D. Diesing, M. Merschdorf, A. Thon and W. Pfeiffer, "Identification of multiphoton induced photocurrents in metal-insulator-metal junctions", Appl. Phys. B-Lasers O. 78(3-4), 443-446 (2004). http://dx.doi. org/10.1007/s00340-003-1391-4 